\title{
3D CAD for Design of Invisible Tooth Aligner
}

\author{
Alan C. Lin, Kuo-Jong Chang, Pai-Ting Wu and Pai-Ei Yih \\ Department of Mechanical Engineering \\ National Taiwan University of Science and Technology \\ Taipei 106, TAIWAN \\ E-mail: alin@mail.ntust.edu.tw
}

\begin{abstract}
Abstract-This paper reports the development of a 3D system for orthodontic treatment of malocclusion tooth occlusion. The CAD system enables the dentist to directly modify a dental model in a computer, output the post-modification file to speedily produce a prototype, and finally fabricate a ready-to-wear invisible tooth aligner. This tool also provides clear evaluation for post orthodontic treatment tracking.
\end{abstract}

\section{INTRODUCTION}

Malocclusion tooth occlusion can be corrected by orthodontic treatment to optimize the occlusal relationship. More and more people choose invisible tooth aligner when they need an orthodontic treatment, as shown in Figure 1 [1-3]. The following summarized the steps of making an invisible tooth aligner [4]:

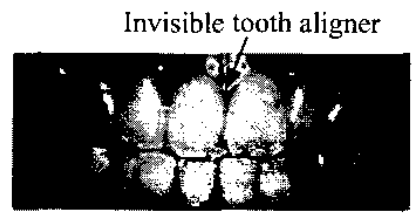

Figure 1 Invisible tooth aligner.

(1) The dentist makes a dental model of the patient's upper and lower tooth alignment.

(2) A dental technician makes a corresponding plaster model.

(3) The tooth which is under treatment is incised off the plaster model manually.

(4) On the plaster model, the dentist manually adjusts the under-treatment teeth to appropriate locations.

(5) An invisible tooth aligner is fabricated using the modified dental model.

(6) Let the patient fit on the invisible tooth aligner and try out.

The ideation springs forth to digitalize the patient's tooth into computer, and let computerized treatment simulation work on the meticulous and toilsome process instead of manual making of plaster prototype to cut production cost and shortens model modification time. The following introduces the process of digitalized production of an invisible tooth aligner:

(1) The dentist makes a dental model of the patient's upper and lower dentition.

(2) A dentistry technician makes a corresponding plaster model.

(3) Use an optical scanner to obtain all data points of the tooth profile from the plaster model.

(4) Use computer to separate the teeth under treatment and proceed with tooth alignment. Output the modified alignment result into STL file.

(5) Use STL file to produce a rapid prototype (RP) model and fabricate an invisible tooth aligner.

(6) Fit the aligner onto the patient and start treatment.

(7) Use computer to calculate the actual tooth movement between before- and after-treatment.

In the above production process, several steps are conducted digitally. The digitalized treatment enables dentists to easily control the current and/or future treatment status, and henceforth to timely modify the therapeutic regime during the treatment. The digitalized orthodontic treatment is explained in the following sections.

\section{OBTAIN The DATA POINTS OF DENTAL MODEL}

This study uses indirect non-contact measurement tool to scan out data points. Figure 2 shows an ATOS optical scanning device used in the experiment. Figure 3 shows the data points during scanning process.

Once 3D data points of the dental model are obtained, proceed to single out and incise each under-treatment tooth. Data points of the digital dental model can be shifted, revolved, displaced, enlarged, and so forth by manipulating the operating window. The data points of the under-treatment tooth can thus be easily sorted out to serve the following orthodontic treatment process.

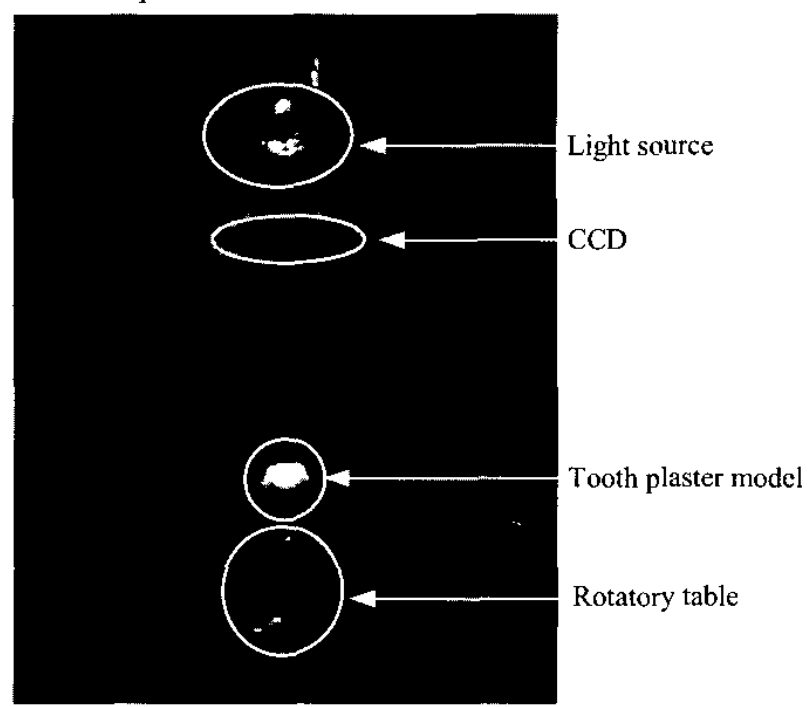

Figure 2. ATOS optical scanning device. 


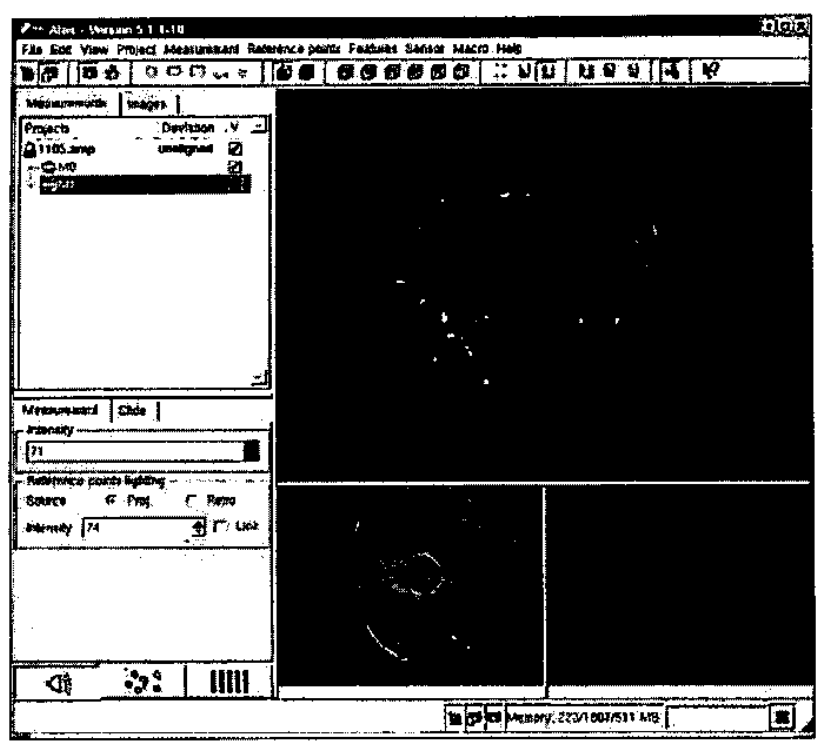

Figure 3. Data points during scanning process.

\section{COMPUTER-AIDED ORTHODONTIC TREATMENT}

\section{A. Movement of the under-treatment tooth}

The movement of the treated is based on the dental arch concept - to achieve orthodontics result by replacing the patient's distorted dental arch with a standard arch (see Figure 4). It starts with marking out both the origin line-segment and the target one. Then calculate the difference between the two lines to be used as the movement value for the under-treatment tooth from as is to the target position. The marked points and lines of the dental arch are shown in Figure 5.

The approximation of the patient's dental arch toward a standard one to well align each tooth's position is divided into two ways: 4-point movement and 6-point movement. Their procedures and objectives are as follows:

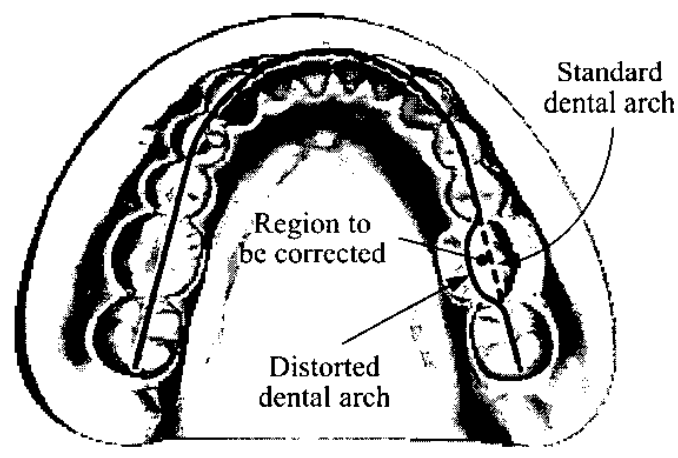

Figure 4. Standard and distorted dental arches.

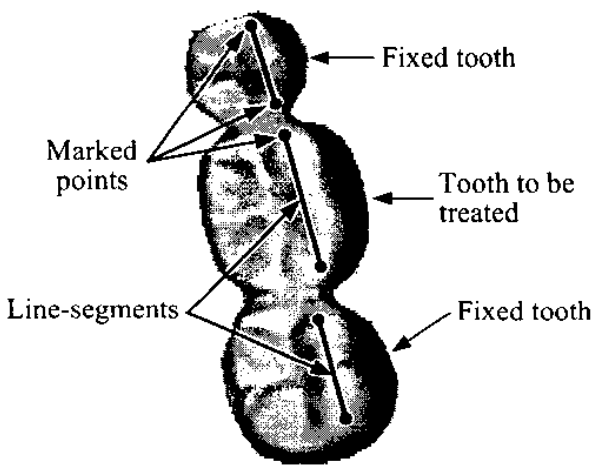

Figure 5. Illustration of marked points and line-segments.

(1) 6-point movement

6-point movement uses two line-segments of a retained tooth as reference to generate a target line-segment. Next, use the under-treatment tooth's dental arc as the origin line-segment and align these two line-segments together. The movement of the line-segment is depicted in Figure 6. However, this method only fits for orthodontic tooth alignment in the lower jaw. The reason is because upper and lower jaws have different dental arches. The dental arch of the upper jaw is not an arc curve like that of the lower jaw, but instead has inflections in the area of canine and first premolar, as shown in Figure 7. Therefore, the 4-point marking method is raised to solve this issue.

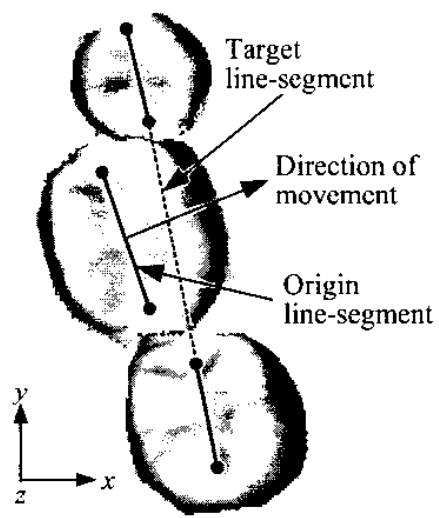

(1) Mark origin and target line-segments

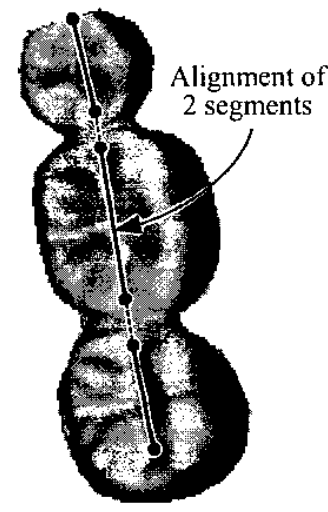

(2) Align origin and target line-segments
Figure 6. Illustration of the 6-point movement method. 


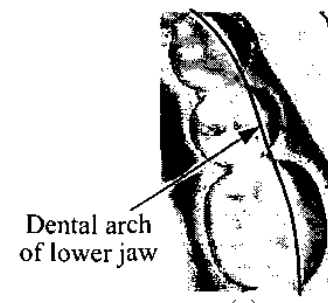

(a)

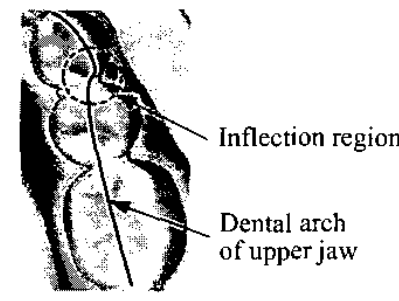

(b)

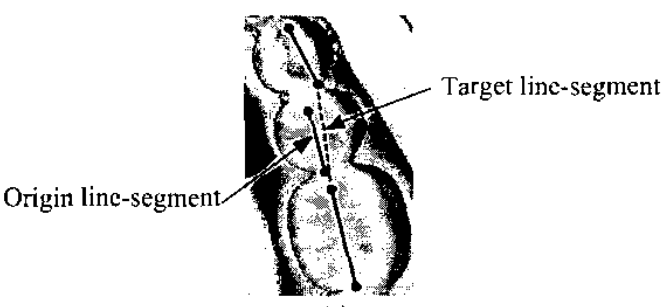

(c)

Figure 7. Limitations of the 6-point movement method.

\section{(2) 4-point movement}

The 4-point movement method is similar to that of 6-point movement, except for the difference that it simply selects four marking points from the user-defined under-treatment tooth as displayed in the monitor and proceed with alignment of the generated two dental arc curves. This method is more flexible than that of 6-point movement, and thus, it contributes to modifications for the 6-point movement in later-on treatment stage. Figure 8 illustrates the tooth movement of this method.

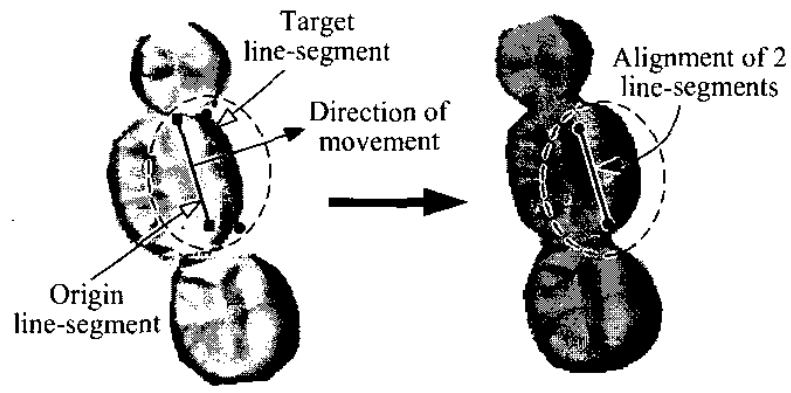

Figure 8. Illustration of the 4-point movement method.

\section{B. Inclination of the under-treatment tooth}

The aforementioned orthodontic treatment is conducted by moving the under-treatment tooth to the desired position on a plane, which is a dual-dimension treatment and solves only for planar misaligned problem. However, there are times when tooth shoves against each other which result in inclination of tooth axis and 3D tooth movement is thus needed, as shown in Figure 9.

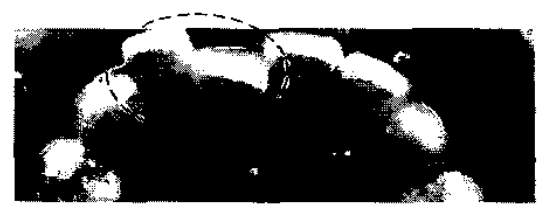

Figure 9. Illustration of an inclined tooth.
The common dental treatment practices against an inclined tooth vary from pure tipping, controlled tipping, to bodily movement and root torque according to dentist's experience and the specific situation. These different methods are briefed in the following (see Figure 10).
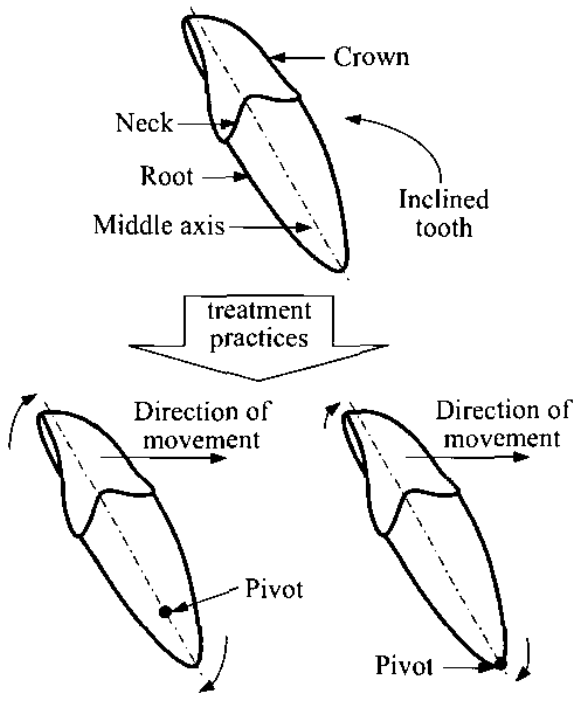

(a) Pure tipping

(b) Controlled tipping
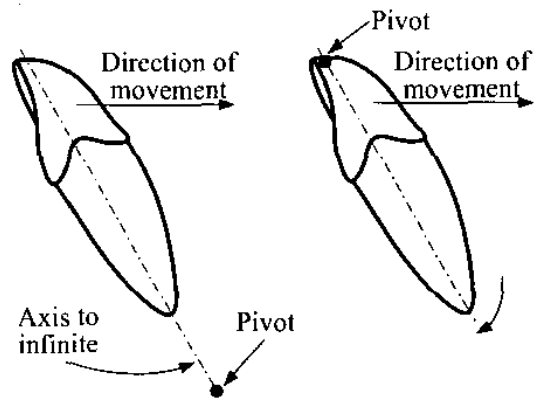

(c) Bodily movement

(d) Root torque

Figure 10. Orthodontic treatment of an inclined tooth.

(1) Pure tipping: As shown in Figure $10(a)$, tooth rotates around a pivot point (or called center of rotation) during the rectification. The pivot point locates is somewhere along the middle axis and above the bottom of the tooth. Moreover, the crown and apical portion of the root tip in the opposite direction.

(2) Controlled tipping: As shown in Figure 10(b), the pivot point locates at the root apex. This method is appropriate when only the crown portion needs to be corrected.

(3) Bodily movement: As shown in Figure $10(c)$, the pivot point locates at infinitum. The tooth moves parallel and no rotation is observed.

(4) Root torque: As shown in Figure 10(d), the pivot point locates at the top of tooth crown. This method is used when only the tooth root needs to be corrected.

Since the tooth root of a patient is non-measurable, the first three of the above four methods are infeasible. To tap such a situation, this study proposes to utilize the middle point and centroid of the 2D dental arch of the under-treatment tooth, in 
conjunction with an estimate of the height of the pivot point to obtain the pivot point for the tooth. As the height of pivot point varies, four orthodontic methods can be further derived as shown in Figure 11.

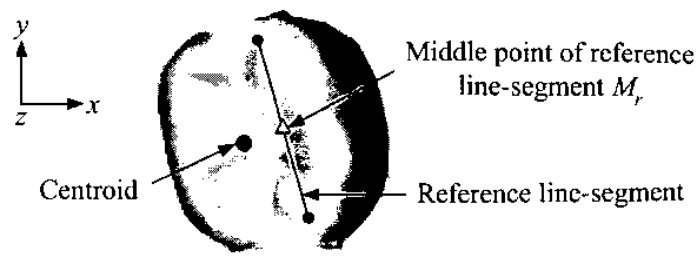

(a) $x$-y plane

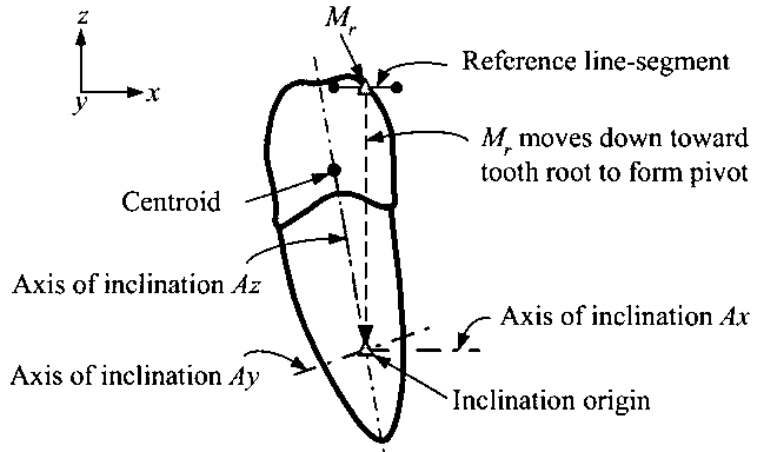

(b) $x-z$ planc

Figure 11, Illustration of finding the pivot point.

\section{Semi-automatic orthodontic treatment}

The so-called semi-automatic orthodontic treatment is to align teeth by utilizing the aforementioned 4-point movement method in conjunction with the concept of "dental arch". Its processes are as follows:

(1) Define feature line-segment of the tooth

Mark the line-segment of every single tooth out of the sectored (and numbered) data points of the dental model. The line-segment refers to the 2D line that represents the tooth's current orientation. This line will serve as a crucial parameter for orthodontics calculation later on (as shown in Figure 12).

(2) Find an appropriate dental arch form

From the user-defined line-segments, derive the tooth parameters that best represent the total length, width of the tooth row and the entire cavity. These parameters are used to find coefficients $a$ and $b$ in the following Beta function $[5,6]$ for forming the dental arch: $y=a \times x^{6}+b \times x^{2}$ (as shown in Figure 13).

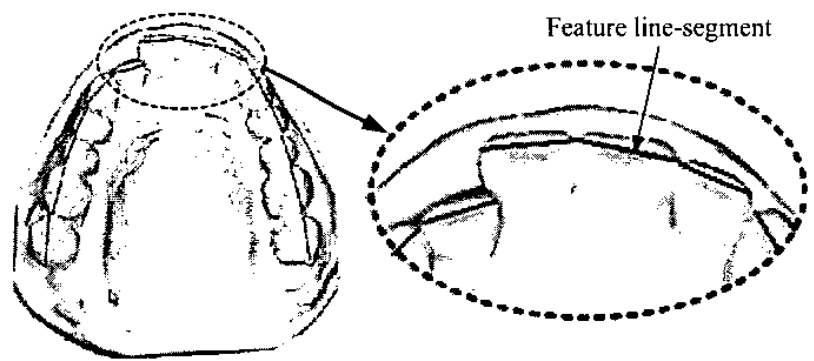

Figure 12. Define Feature line-segments for all teeth.

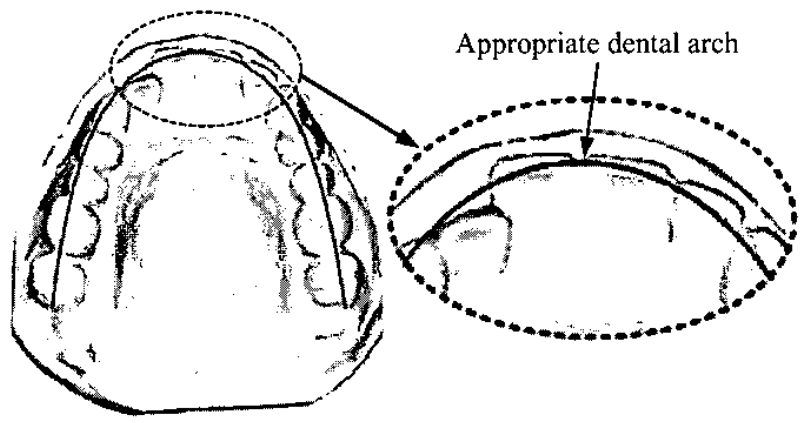

Figure 13. Find an appropriate dental arch form.

(3) Conduct automatic tooth alignment

After obtaining the dental arch form, find the target line-segment of each tooth by its sequence order on the arch. The line-segments located on each of the treatment teeth are set as the origin line-segments. Then the origin line-segments are aligned with target line-segments as shown in Figure 14.

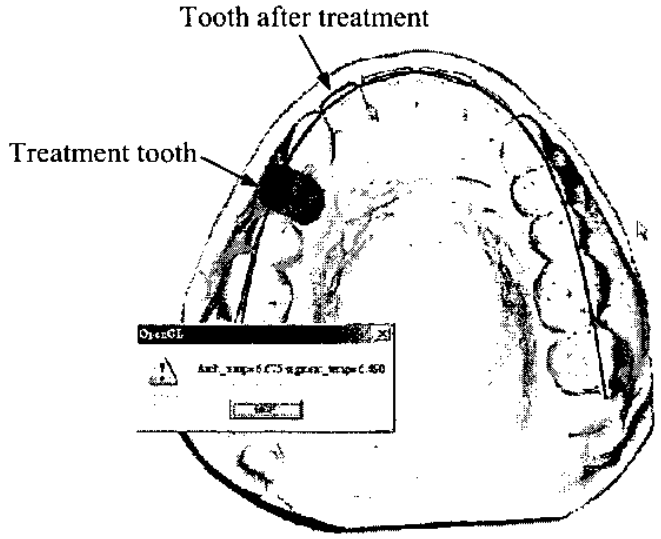

Figure 14. Illustration of automatic tooth-aligning treatment.

\section{FABRICATION OF INVISIBLE TOOTH ALIGNER}

After a series of tooth treatment process, the resultant model is output in STL file, and an RP model is constructed by using an Object Quadra Tempo rapid prototyping machine as shown in Figure 15. Replicating the RP model by vacuum-heat processing of transparent polymers, as shown in Figure 16, forms a thin-shell invisible tooth aligner.

\section{Conclusions}

The objective of this study sets out to contribute effective application of digital tools to the treatment process of invisible tooth aligner. It is much hoped that, with the aid of digital simulation, high-tech rapid prototyping and accurate measurements in every step of treatment, dentists may fully concentrate on orthodontic treatment per se, and be spared from meticulous toil. Dentists can also clearly control the patient's treatment status at every stage. The digitalized orthodontic treatment process is beneficial for dentists to excel in professional treatment presentation and therapeutic diagnosis, while saving more time for patients to interact with dentists for a better understanding of their current and future treatment status. 
(a) RP machine

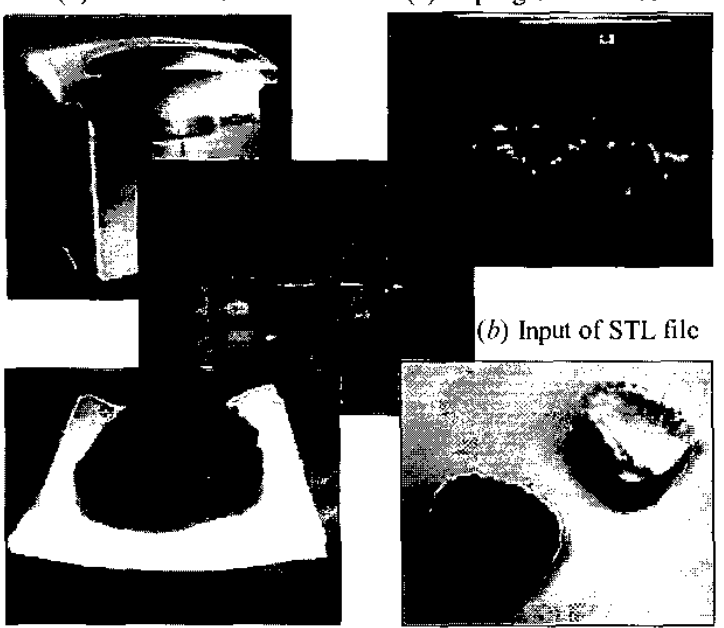

(d) Completed RP model (e) RP model and plaster model
Figure 15. Procedure of making an RP model.

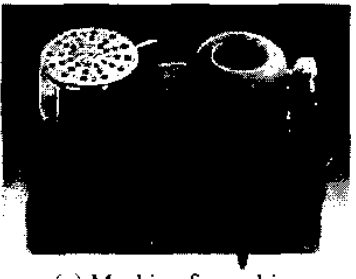

(a) Machine for making invisible tooth aligner

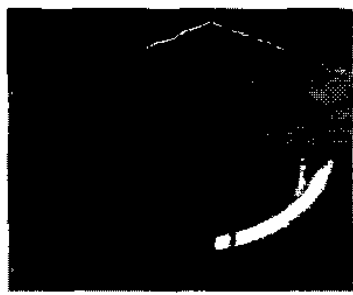

(c) After vacuum-heat processing

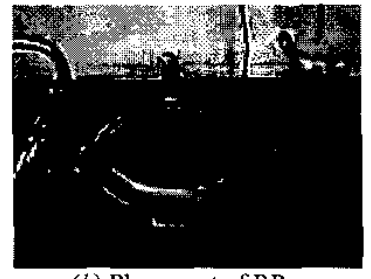

(b) Placement of RP model in steel sand

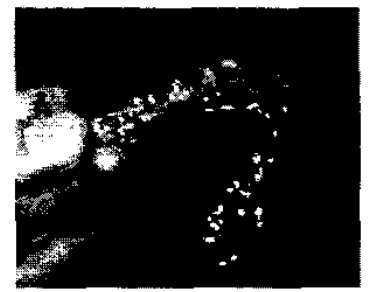

(d) Invisible tooth aligner after trimming the contour
Figure 16. Procedure of making the invisible tooth aligner.

\section{REFERENCES}

[1] Y.J. Chen, H.F. Chang, H.C. Tsai, and C.C. Yao, "Orthodontic Correction of Upper Incisors Crowding Using the Invisalign System - Case Report", Chinese Dental Journal, Vol. 4, pp. 1-10, 2003.

[2] Z.C. Chang, C.C. Yao, H.F. Chang, Y.J. Chen, "Clinical Application of Customized Removable and Clear Orthodontic Aligners - Case Report", Chinese Dental Journal, vol. 22, no. 4, pp. 363-370, 2003.

[3] Y.J. Chen, K.H. Chen, S.K. Chen, C.C. Yao, H.F. Chang, "A Survey on the NTUH Orthodontic Patients' Attitude Toward Invisible Removable Aligner", Chinese Dental Journal, 2004 (in press).

[4] H.D. Kesling, "The Philosophy of Tooth Positioning
Appliances", American Journal of Orthodontics, vol. 31, pp. 297-304, 1945.

[5] H. Noroozi, T.H. Nik, R. Saeeda, "The Dental Arch From Revisited", Angle of Orthodontics, vol. 71, no. 5, pp. 386-389, 2001.

[6] S. Braun, W.P. Hnat, D.E. Fender, H.L. Legan, "The Form of the Human Dental Arch", Angle of Orthodontics, vol, 68 , no. 10, pp. 29-36, 1998.

\section{ACKNOWLEDGEMENTS}

This research was supported in part by the National Science Council, Taiwan, through Grant No. NSC 90-2212-E-011-050, NSC 91-2212-E-011-023, and NSC 92-2212-E-011-026. 